\title{
Biomechanical effects of osteoplasty with or without Kirschner wire augmentation on long bone diaphyses undergoing bending stress: implications for percutaneous imaging-guided consolidation in cancer patients
}

Roberto Luigi Cazzato ${ }^{1 * \dagger}$ (D), Guillaume Koch ${ }^{1+}$, Julien Garnon ${ }^{1}$, Nitin Ramamurthy², Jérémie Jégu ${ }^{3}$, Philippe Clavert ${ }^{4}$ and Afshin Gangi ${ }^{1}$

\begin{abstract}
Background: Osteoplasty has been discouraged in long bones. However, despite a substantial lack of pre-clinical biomechanical tests, multiple clinical studies have implemented a wide range of techniques to optimise long bone osteoplasty. The aim of the present study is to evaluate the biomechanical properties of osteoplasty alone and in combination with Kirschner wires (K-wires) in a cadaveric human diaphyseal model undergoing 3-point bending stress.

Methods: Thirty unpaired human cadaveric hemi-tibia specimens were randomly assigned to receive no consolidation (group 1, $n=10$ ), osteoplasty alone (group $2, n=10$ ), or K-wires augmented osteoplasty (group 3 , $n=10$ ). Specimens were tested on a dedicated servo-hydraulic machine using a 3-point bending test. Fracture load was calculated for each specimen; two-sample Wilcoxon rank-sum tests were used to assess differences between groups.
\end{abstract}

Results: Median volume of polymethyl methacrylate injected was $18 \mathrm{~mL}$ for group 2 (25th-50th percentile $15-21 \mathrm{~mL}$ ) and $19 \mathrm{~mL}$ for group 3 (25th-50th percentile 17-21). There were no significant differences in fracture load between groups 1 and $2(z=-0.793 ; p=0.430)$, between groups 1 and $3(z=-0.944 ; p=0.347)$, and between groups 2 and 3 $(z=-0.454 ; p=0.650)$. Fractures through the cement occurred in 4 of 30 cases $(13.3 \%)$; there were no K-wires fractures.

Conclusions: Osteoplasty with or without K-wires augmentation does not improve the resistance of diaphyseal bone to bending stresses.

Keywords: Bone wires, Diaphyses, Fractures (bone), Polymethyl methacrylate

\footnotetext{
* Correspondence: gigicazzato@hotmail.it

${ }^{\dagger}$ Roberto Luigi Cazzato and Guillaume Koch contributed equally to this work.

'Department of Interventional Radiology, Nouvel Hôpital Civil (Hôpitaux

Universitaires de Strasbourg, HUS), 1, place de l'Hôpital, 67000 Strasbourg,

France

Full list of author information is available at the end of the article
} 


\section{Key points}

- Osteoplasty is discouraged for long bone tumours due to the risk of secondary fractures.

- Multiple clinical studies have implemented a wide range of techniques to optimise long bone osteoplasty without pre-clinical biomechanical assessment.

- The biomechanical properties of osteoplasty with or without Kirschner wires augmentation have been tested in long bones.

- Osteoplasty with or without Kirschner wires augmentation did not improve the biomechanical properties of long bones.

\section{Background}

Bone-seeking tumours (lung, breast, renal, prostate, and multiple myeloma) account for $45 \%$ of commonly diagnosed cancers [1]. Metastatic tumours typically occur in the spine, pelvis, and proximal femur and are complicated by pathological fracture in 17 to $43 \%$ of patients [2]. Pathological/impending fractures in long bones are particularly associated with advanced disease [3, 4], severe pain, functional loss, and devastating consequences on quality of life (QoL) and prognosis [3]. Prompt stabilisation is essential to optimise the outcome.

Surgical fixation provides superior construct durability, but is frequently unsuitable for frail oncologic patients, and may negatively impact QoL and survival [5]. Recently, the development of percutaneous imaging-guided therapies including osteoplasty and screw fixation has provided a minimally invasive, effective, and safe alternative to surgery in cancer patients. However, despite excellent palliative results following the consolidation of long bone tumours, osteoplasty alone is associated with frequent secondary long bone fractures, occurring in approximately $8 \%$ of cases [6]. This has been attributed to suboptimal material properties of polymethyl methacrylate (PMMA) cement, weaker under bending stress $(64 \mathrm{MPa})$ than under compression (93 MPa); and osteoplasty alone has been discouraged for long bone tumours [7, 8]. Multiple clinical studies have recently implemented a wide range of adjunctive techniques to optimise long bone consolidation [9-18] (Table 1). However, pre-clinical biomechanical studies are substantially lacking.

The purpose of this study was to evaluate the biomechanical properties of osteoplasty alone and in combination with Kirschner wires (K-wires) in a cadaveric human diaphyseal model subjected to 3-point bending stress as well as to discuss implications for percutaneous imaging-guided consolidation of long bone metastases.

\section{Methods}

Specimens were harvested in compliance with institutional safety regulations. Institutional review board approval was obtained for this cadaveric study in the setting of routine research activity on human cadavers performed at the University Hospital of Strasbourg.

Table 1 Published studies reporting on different consolidative techniques in long bones

\begin{tabular}{|c|c|c|c|c|c|c|}
\hline $\begin{array}{l}\text { First author } \\
\text { [reference] }\end{array}$ & Journal & Year & $\begin{array}{l}\text { Number of } \\
\text { patients }\end{array}$ & Target bone & Type of intervention & $\begin{array}{l}\text { Secondary } \\
\text { fractures (\%) }\end{array}$ \\
\hline Cazzato [8] & Eur Radiol & 2014 & 51 & Long bones & Osteoplasty & 9.1 \\
\hline Premat [9] & Eur Radiol & 2017 & 18 & $\begin{array}{l}\text { Proximal } \\
\text { femur }\end{array}$ & Spindles + osteoplasty & 0 \\
\hline Kelekis [10] & $\mathrm{CVIR}$ & 2016 & 12 & Long bones & 25-50 stainless steel micro-needles + osteoplasty & 0 \\
\hline \multirow[t]{2}{*}{ Liu [11] } & \multirow[t]{2}{*}{ Eur Radiol } & \multirow[t]{2}{*}{2016} & \multirow[t]{2}{*}{36} & \multirow[t]{2}{*}{ Long bones } & Osteoplasty (19 patients) & 26.3 \\
\hline & & & & & $\begin{array}{l}\text { Osteoplasty + cement-filled catheter in the medullary } \\
\text { canal ( } 17 \text { patients) }\end{array}$ & 0 \\
\hline \multirow[t]{2}{*}{ Tian [12] } & \multirow[t]{2}{*}{ CVIR } & \multirow[t]{2}{*}{2014} & \multirow[t]{2}{*}{40} & \multirow{2}{*}{$\begin{array}{l}\text { Proximal } \\
\text { femur }\end{array}$} & Osteoplasty (19 patients) & 23.8 \\
\hline & & & & & $\begin{array}{l}\text { Osteoplasty + internal fixation with bone trocars stylets } \\
(21 \text { patients) }\end{array}$ & 0 \\
\hline $\mathrm{He}[13]$ & $J V I R$ & 2014 & 6 & $\begin{array}{l}\text { Proximal } \\
\text { femur }\end{array}$ & Osteoplasty + internal fixation (bone trocars stylets) & 0 \\
\hline Cazzato [14] & Eur J Radiol & 2017 & 11 & $\begin{array}{l}\text { Proximal } \\
\text { femur }\end{array}$ & Osteosynthesis & 0 \\
\hline Lin [15] & Surg Oncol & 2015 & 12 & $\begin{array}{l}\text { Proximal } \\
\text { femur }\end{array}$ & $\begin{array}{l}\text { Osteosynthesis with modified hollow-perforated screws } \\
\text { and osteoplasty }\end{array}$ & 8.3 \\
\hline Cornelis [16] & $\begin{array}{l}\text { J Orthop Surg } \\
\text { Res }\end{array}$ & 2017 & 10 & $\begin{array}{l}\text { Proximal } \\
\text { femur }\end{array}$ & Y-STRUT ${ }^{\oplus}$ device & 10 \\
\hline Kim [17] & Surg Oncol & 2011 & 15 & Humerus & Ender nail fixation and osteoplasty & NR \\
\hline Kim [18] & Surg Oncol & 2014 & 15 & $\begin{array}{l}\text { Femur and } \\
\text { tibia }\end{array}$ & Flexible nailing and osteoplasty & NR \\
\hline
\end{tabular}




\section{Bone specimens and study sample}

Ten pairs of embalmed human cadaveric tibias were obtained from five cadavers (three male, two female, mean age 75 years, range 67-93 years; mean height $1.67 \mathrm{~m}$, range $1.52-1.78 \mathrm{~m}$ ) donated to the institutional Anatomy Department of the University Hospital of Strasbourg and preserved by injection of formalin and alcohol solution into the femoral artery. There were no cases of previous surgery or anatomic alteration to the lower legs. Specimens were stored in $20 \%$ alcohol solution prior to application and kept moist throughout the study using intermittent saline irrigation.

Each tibia was measured in long axis and axially transected in the mid-diaphysis using an oscillating bone saw. A total of 40 experimental specimens (proximal/distal, right/left hemi-tibias) were obtained. Ten randomly selected specimens were utilised for initial feasibility assessment and excluded from the final study sample. Six were used to assess the technical feasibility of osteoplasty and K-wires augmented osteoplasty procedures and to optimise biomechanical loading protocols. Four specimens were used to assess the technical feasibility of osteoplasty with PMMA-filled catheter augmentation, a novel procedure in which PMMA-filled biliary catheters are used as intramedullary nails to optimise the consolidative properties of PMMA osteoplasty [11]. The technique utilises accessible and familiar equipment and may provide clinical benefits [11]. Unfortunately, it was not possible to replicate this construct in our cadaveric specimens. Despite numerous attempts to manually inject PMMA (using 3-mL syringes at room temperature of $22^{\circ} \mathrm{C}$ ) into $8-14$ Fr biliary catheters positioned longitudinally within the bone specimens using a vertebroplasty trocar $(n=4)$ and extra-osseously $(n=1)$, it was not possible to obtain homogeneous intraluminal cement distribution without causing catheter fracture. This method was therefore not investigated.

The final study sample comprised 30 specimens. Computed tomography (CT) was performed $(90 \mathrm{mAs}$; $120 \mathrm{kVp}$ ) to exclude pre-existing focal disease and estimate cortical bone density using $\mathrm{x}$-ray attenuation values (mean of three $1-\mathrm{mm}^{2}$ regions of interest placed in epiphyseal, metaphyseal, and diaphyseal cortex on axial 1-mm slices). Formal bone mineral density estimation and geometric analysis were precluded by the unavailability of peripheral quantitative CT or dual-energy $\mathrm{x}$-ray absorptiometry.

Bone specimens were randomly assigned to three groups: no consolidative therapy (group1), osteoplasty alone (group 2), and K-wires augmented osteoplasty (group 3) (Fig. 1). Intra-individual controlled pair matching could not be performed due to the paucity of specimens.

\section{Osteoplasty and K-wires augmentation}

Two interventional radiologists with 3- and 5-year experience in bone consolidation performed bone augmentation (groups 2 and 3). For group 2, osteoplasty was performed using a $10-\mathrm{G}$ vertebroplasty needle (Gangi Special Vertebroplasty Needle Set, Optimed, Ettlingen, Germany) advanced within the central long axis of the specimen via the transected surface. Under continuous fluoroscopy, PMMA cement (Osteopal V, Heraeus medical, Wehrheim, Germany) was manually injected using $5-\mathrm{mL}$ syringes until leakage occurred or no further cement could be injected. For group 3, three $\mathrm{K}$-wires were sequentially advanced through the cut bone surface using an electric driver and positioned paramedian to the long axis of the diaphysis in a triangular configuration. Osteoplasty was then performed as above.

\section{Biomechanical testing and fracture assessment}

Due to the limited number of specimens available, only 3-point bending tests were conducted on a dedicated servo-hydraulic machine (INSTRON 8500 plus, INSTRON Corporation, High Wycombe, Buckinghamshire, UK). Although the 3-point bending test may be influenced by shearing stresses, the choice to apply such test was justified by the fact that beams undergoing axial load such as long bones of the lower limbs are also subjected to bending in a direction that is perpendicular to that of the applied axial load (i.e., buckling).

Specimens were positioned horizontally on two fixed supports, lateral aspect facing downwards; the distance

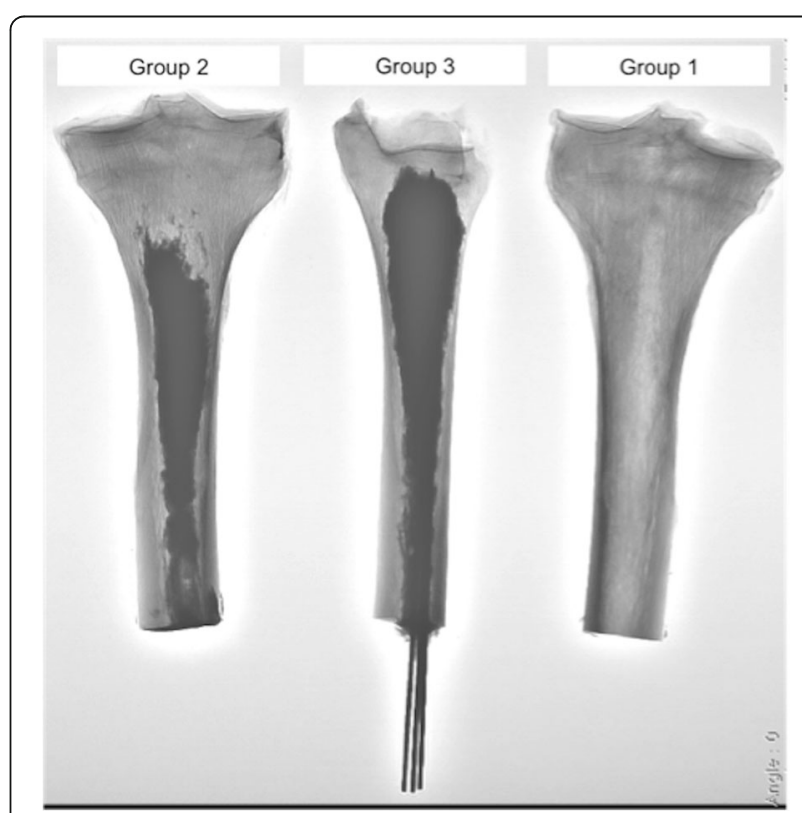

Fig. 1 Fluoroscopic image illustrating a bone sample from each experimental group 
between the two points was fixed. A 10-N pre-load was applied midway between the two supports via a metal wedge impact anvil attached to the load cell (Fig. 2). The machine actuator was displaced inferiorly at $50 \mathrm{~mm} / \mathrm{s}$ until the specimen fractured, while force/displacement data were recorded at $2,500 \mathrm{~Hz}$. The final displacement of the actuator was measured by assuming that the elastic deformation of the machine was negligible compared to that of tested specimens.

Data were transferred to an Excel spreadsheet (Microsoft Corporation, Redmond, WA, USA), force/ displacement curves were plotted for each group, and fracture load was automatically calculated by proprietary software. Data regarding Young's modulus were also provided.

Fractures involving bone and/or consolidative constructs were documented using two orthogonal plain radiographs. Location and morphology were classified in consensus by two board-certified radiologists (3- and 5 -year experience), according to a modified version of the long bone fracture classification of the Müller AO Trauma Foundation [19], in which wedge fractures not extending to the opposite cortex were additionally labelled as $B O$ (Fig. 3).

\section{Data collection and statistics}

Anatomic origin, length, mean cortical CT attenuation, quantity of PMMA injected, fracture load, Young's modulus, and type of fracture involving bone/constructs were recorded for each specimen. Results were presented using descriptive statistics, and two-sample Wilcoxon rank-sum test was used to compare fracture load between groups. All statistical analysis was performed with Matlab $^{\circ}$ (MathWorks, Inc., Natick, MA, USA); $p$ values lower than 0.05 were considered significant.

\section{Results}

Specimen anatomic origin, median length, and median estimated cortical CT attenuation value for each group are summarised in Table 2. Median volume of PMMA injected was $18 \mathrm{~mL}$ for group 2 (25th-50th percentile $15-21 \mathrm{~mL}$ ) and $19 \mathrm{~mL}$ for group 3 (25th-50th percentile $17-21 \mathrm{~mL}$ ).

Force/displacement curves for each group are provided in Fig. 4. Fracture load was numerically higher in consolidated groups than in control specimens (Table 3). However, there was no significant difference between groups 1 and $2(z=-0.793 ; p=0.430)$, between groups 1 and $3(z=-0.944 ; p=0.347)$, and between groups 2 and $3(z=-0.454 ; p=0.650)$.

The most common type of fracture was the $B O(14 / 30$, $46.6 \%)$ and the $A 2(8 / 30,26.6 \%)$ (Table 4). Fractures through the cement occurred in 4 of 30 cases (10\%), including one from group 2 and three from group 3 . There were no K-wires fractures in group 3.

Young's modulus did not differ significantly between groups 1 and $2(z=0.121 ; p=0.904)$, between groups 1 and $3(z=0.338 ; p=0.728)$, and between groups 2 and 3 $(z=0.148 ; p=0.881)$ (Table 3$)$.

\section{Discussion}

Despite the clinical inadequacy of stand-alone osteoplasty for consolidation of long bone pathological/ impending fractures [6] and the rapid implementation of numerous alternative techniques [9-18], there has been little evaluation of the biomechanical efficacy of these procedures. In the proximal femur, a few cadaveric studies have shown that femoroplasty effectively consolidates osteoporotic bone subjected to sideways falling [20-22] and may reduce mechanical stress around stance-loaded simulated femoral neck tumours provided that cement filling is adequate [23]. Similarly, the novel Y-STRUT ${ }^{\circ}$

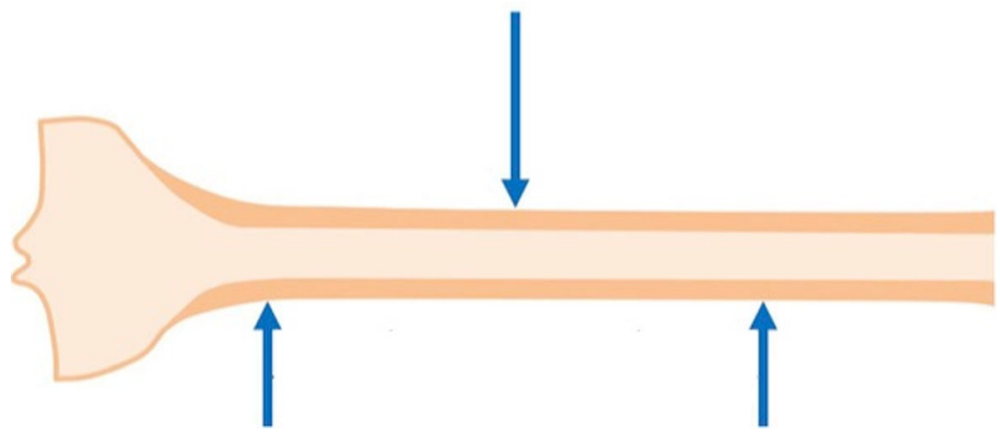

Fig. 2 Schematic representation of the 3-point bending test protocol. $F$ force 


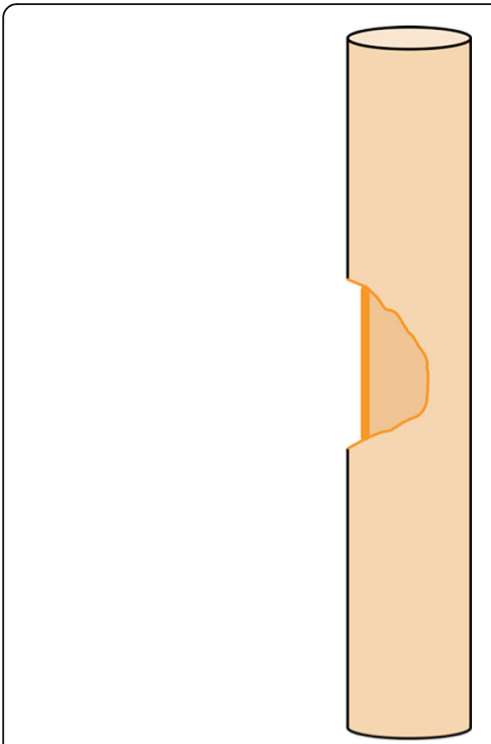

Fig. 3 Schematic representation of a $B O$ fracture type according to a modified Müller $\mathrm{AO}$ classification used in the present study

implant (Hyprevention, Pessac, France; designed to prevent hip fracture) has undergone pre-clinical validation, demonstrating reduced fracture risk during sideways falling [24]. In contrast, there are only three prior animal studies evaluating the biomechanical effects of diaphyseal augmentation. These illustrated a lower bending strength of osteoplasty alone than when combined with bare metal stents or $\mathrm{K}$-wires, in porcine and bovine models with simulated diaphyseal fractures and focal tumours [25-27].

The present study demonstrates that osteoplasty alone or with K-wires augmentation does not confer any consolidative advantage to diaphyseal bone undergoing 3 -point bending stress. Fracture load was similar to controls for both composites, consistent with the lack of consolidative and stiffening effects. The stiffness of the tested specimens was slightly increased in composites from group 2 and group 3. Nevertheless, there was no significant difference as compared to controls.

There were four PMMA fractures, consistent with the brittle nature of PMMA and its unsuitability to resist non-compressive loads.
Multiple alternative constructs have been proposed to optimise the consolidation of oncologic long bone tumours, particularly in the proximal femur, with relatively few series treating diaphyseal tumours (only $6 \%$ of cases in a recent systematic review [6]).

In several reports, osteoplasty has been combined with dedicated spindles, modified mandrins, and multiple micro-needle mesh to optimise biomechanical resistance of PMMA to multi-directional stresses (rebar concept) [10]. Studies in the proximal femur $[9,10,12]$ and long bone diaphyses [10] have illustrated good analgesia and restoration of functional status, with either no secondary fractures $[9,10]$ or fewer than with osteoplasty alone [12], at 6-16-month follow-up. Our study did not demonstrate any beneficial effect of osteoplasty augmented with K-wires, although this may reflect sample limitations, test protocol, or suboptimal composite material properties. Nevertheless, there remains a lack of biomechanical evidence and long-term follow-up to support these procedures.

Other studies have adapted surgical techniques to improve long bone tumour stabilisation. In the proximal femur, percutaneous screw fixation (simulating the inverted triangle configuration of orthopaedic procedures) $[14,15,17]$ and placement of the Y-STRUT ${ }^{\circ}$ device simulating a gamma nail [16] have been implemented to treat pathological/impending fractures in selected non-surgical patients. Early results are encouraging, although secondary fracture rates remain considerable (from 6 to 10\%) [13, 16]. In contrast, long bone diaphyseal fixation constructs have been largely improvised using interventional radiology equipment. PMMA-filled catheters [11] have been used to simulate the load-sharing action of intramedullary nails (IMN) and augmented osteoplasty of impending fractures. In the series of Liu et al. [11], this resulted in improved analgesia, functional status, and reduced secondary fractures compared with osteoplasty alone. Unfortunately, we were unable to evaluate and replicate this procedure in vitro-possibly due to lower ambient temperature, PMMA viscosity differences, and impedance of PMMA flow by the narrow catheter tip and luminal plugging with trabecular bone rather than tumour. Currently, there is insufficient biomechanical and clinical data to support these techniques.

Table 2 Baseline characteristics of the experimental specimens

\begin{tabular}{lllllll}
\hline $\begin{array}{l}\text { Group } \\
\text { inferior } \\
\text { specimens }\end{array}$ & $\begin{array}{l}\text { Number of } \\
\text { superior } \\
\text { specimens }\end{array}$ & $\begin{array}{l}\text { Number of right } \\
\text { specimens }\end{array}$ & $\begin{array}{l}\text { Number of left } \\
\text { specimens }\end{array}$ & $\begin{array}{l}\text { Median specimen length (cm, } \\
\text { 25th-75th percentile) }\end{array}$ & $\begin{array}{l}\text { Median specimen density (HU, } \\
\text { 25th-75th percentile) }\end{array}$ \\
\hline 1 & 8 & 2 & 6 & 4 & $18.375(17.9-19.25)$ & $1621(1571-1652)$ \\
2 & 4 & 6 & 6 & 4 & $18.375(17.6-19.25)$ & $1600(1550-1613)$ \\
3 & 5 & 5 & 3 & 7 & $18.325(18-19.25)$ & $1606.5(1550-1632)$ \\
\hline
\end{tabular}

HU Hounsfield units 
Cazzato et al. European Radiology Experimental

(2019) 3:4

Page 6 of 9
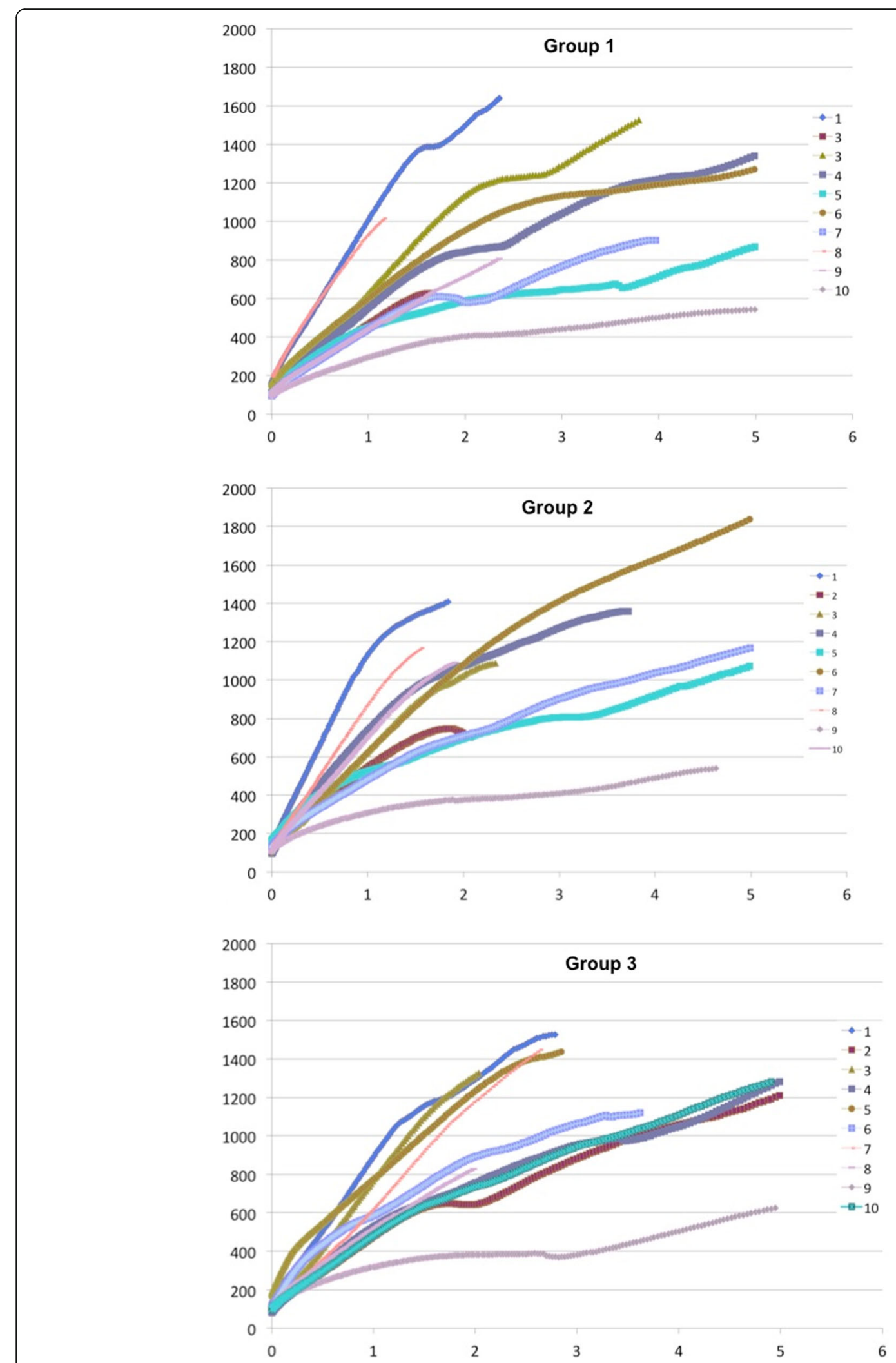

Fig. 4 Force/displacement curves. $x$-axis reports specimen displacement $(\mathrm{mm})$; $y$-axis reports the fracture loading force (N). N Newton 
Table 3 Fracture load and Young's modulus across experimental groups

\begin{tabular}{|c|c|c|c|c|}
\hline \multirow[t]{2}{*}{ Group } & \multicolumn{2}{|l|}{ Fracture load (N) } & \multicolumn{2}{|c|}{ Young's modulus $\left(\mathrm{N} / \mathrm{m}^{2}\right)$} \\
\hline & Mean (SD) & Median (25th-75th percentile) & Mean (SD) & Median (25th-75th percentile) \\
\hline 1 & $1077.6(370.16)$ & $1076(807-1341)$ & $397.15(140.07)$ & $361.39(300.65-514.07)$ \\
\hline 2 & $1221.5(338.12)$ & $1166(1091-1391)$ & $444.53(153.48)$ & $497.88(309.77-556.35)$ \\
\hline 3 & $1230(292.58)$ & $1280.5(1119-1448)$ & $430.73(140.14)$ & $392.23(344.59-547.15)$ \\
\hline
\end{tabular}

$N$ Newton, SD standard deviation

The most promising techniques for diaphyseal tumoural consolidation are probably flexible and bundle IMN (Fig. 5); these have been translated from orthopaedic practice to pathological/impending fractures in cancer patients $[17,18]$ and are already supported by substantial biomechanical and clinical evidence [28, 29]. Flexible IMN produces symmetrical bracing 3-point fixation and achieves (in combination with muscular action) dynamic multi-directional stability. Bundle IMN produces a similar effect via placement of multiple intramedullary pins until the medullary cavity is filled and there is tight compression between the nails and bone [30]. Constructs are typically augmented with PMMA to improve load-sharing and load-bearing properties. Initial clinical results have been promising. Kim et al. [18] performed osteoplasty with flexible IMN in 15 lower limb impending fractures and reported significant palliation, restoration of mobility, and local tumour control (reduction in standardised uptake value at positron emission tomography CT) at 6-month follow-up. Similarly, positive outcomes were also reported for humeral tumours [17]. Disadvantages of the technique include reduced load-sharing properties, lower resistance to torsional/ bending stresses [28], and telescoping, compared with standard surgical rigid IMN with proximal/distal interlocking screw fixation [31, 32]. Further studies with long-term follow-up are required to assess these promising interventions.

Our study limitations are mainly related to unavailability of cadaveric specimens. Therefore, neither additional constructs (e.g., flexible, bundle IMN) nor other possible stresses such as torsion or axial load were evaluated. Moreover, our model did not include a bone defect simulating a diaphyseal bone tumour. However, the present study aimed at investigating the biomechanical

Table 4 Type of fractures according to the modified Müller AO Trauma Foundation Long bone Fracture classification (Reference [19])

\begin{tabular}{lllllllllllll}
\hline Group & A0 & A1 & A2 & A3 & B0 & B1 & B2 & B3 & C0 & C1 & C2 & C3 \\
\hline 1 & 0 & 2 & 0 & 1 & 6 & 0 & 0 & 1 & 0 & 0 & 0 & 0 \\
2 & 0 & 0 & 4 & 1 & 5 & 0 & 0 & 0 & 0 & 0 & 0 & 0 \\
3 & 0 & 0 & 4 & 2 & 3 & 0 & 0 & 1 & 0 & 0 & 0 & 0 \\
Total & 0 & 2 & 8 & 4 & 14 & 0 & 0 & 2 & 0 & 0 & 0 & 0 \\
\hline
\end{tabular}

advantage of osteoplasty or K-wires osteoplasty as compared to the native condition of the target bone. Given the results obtained, one may speculate that similar conclusions might be probably expected also in a model including the same bone defect in all the tested specimens of the three groups. Furthermore, it was not possible to assess bone mineral density and geometric measurements due to unavailability of quantitative CT or dual-energy $\mathrm{x}$-ray absorptiometry or utilise intra-individual controls. However, the majority of fracture risk is accounted for by cross-sectional area of bone rather than mineralisation or morphology [33], and there may be a wide intra-individual heterogeneity even in matched cadaveric samples [34]. The test protocol could have influenced the results in terms of anisotropic effects (varus stress only) and use of hemi-tibias rather than complete bones. However, the choice of 3-point bending test rather than 4-point bending test was made to evaluate the bone strength in the direction

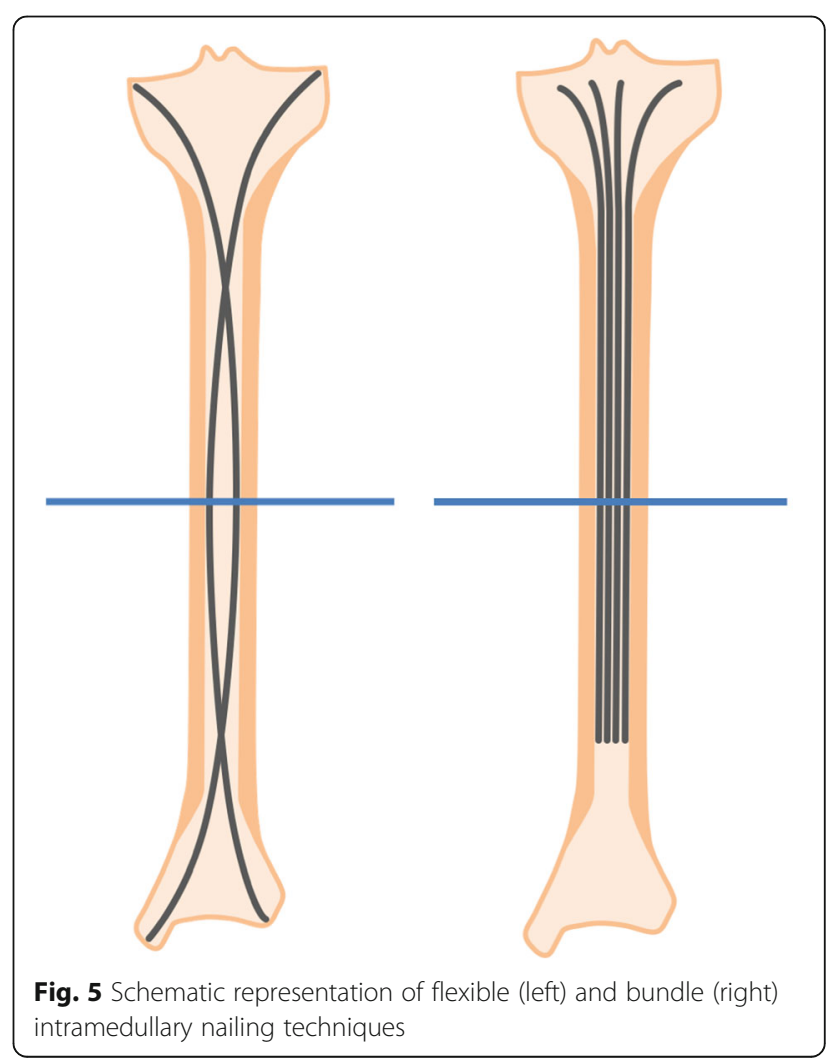


perpendicular to the compression axis. As a matter of fact, long bones (especially in the lower limbs) subjected to axial loads can break due to buckling. Finally, 93\% of fractures (mainly $A 2$ and $B O$ types) were entirely consistent with a bending mechanism, and spiral $A 1$ (torsional) pattern was seen in only two cases (7\%), suggesting reasonable biomechanical reproducibility.

In conclusion, this study confirms that osteoplasty alone or in combination with K-wires does not improve the resistance of diaphyseal bone subjected to bending stress. Therefore, at the moment, long bone osteoplasty and its variants should still be considered as a suboptimal choice for the consolidation of pathological/impending diaphyseal fractures; as a result, this technique should be avoided in good-prognosis cancer patients and proposed with caution in poor-prognosis, predominantly bed-ridden cancer patients presenting with painful lytic tumours.

\section{Abbreviations}

CT: Computed tomography; IMN: Intramedullary nails; K-wires: Kirschner wires; PMMA: Polymethyl methacrylate; QoL: Quality of life

\section{Acknowledgements}

Authors thank Mr. Philippe MAXY for his assistance in conceiving and writing this article.

\section{Funding}

This work received no funding.

\section{Availability of data and materials}

The relevant data have been included in the manuscript. The datasets used and/or analysed during the current study are available from the corresponding author on reasonable request.

\section{Authors' contributions}

RLC, GK, and AG conceived the study. RLC, GK, JG, PC, and AG collected the data. RLC, GK, JG, PC, JJ, and AG analysed and interpreted the data. RLC, GK, and NR drafted the paper. All authors critically revised the manuscript. All authors read and approved the final manuscript.

\section{Ethics approval and consent to participate}

Institutional review board approval was obtained for this cadaveric study in the setting of routine research activity on human cadavers performed at the University Hospital of Strasbourg.

\section{Consent for publication}

Not applicable.

\section{Competing interests}

The authors declare that they have no competing interests.

\section{Publisher's Note}

Springer Nature remains neutral with regard to jurisdictional claims in published maps and institutional affiliations.

\footnotetext{
Author details

'Department of Interventional Radiology, Nouvel Hôpital Civil (Hôpitaux Universitaires de Strasbourg, HUS), 1, place de l'Hôpital, 67000 Strasbourg, France. ${ }^{2}$ Department of Radiology, Norfolk and Norwich University Hospital, Colney Lane, Norwich NR4 7UY, UK. 'Laboratoire d'Epidémiologie et de Santé Publique - EA3430, Université de Strasbourg, 4, Rue Kirschleger, 67085 Strasbourg, France. ${ }^{4}$ Department of Normal Anatomy, Hôpitaux Universitaires de Strasbourg, HUS France, 1, place de I'Hôpital, 67000 Strasbourg, France.
}

Received: 31 October 2018 Accepted: 14 December 2018

Published online: 28 January 2019

\section{References}

1. Kelly M, Lee M, Clarkson P, O'Brien PJ (2012) Metastatic disease of the long bones: a review of the health care burden in a major trauma centre. Can J Surg 55:95-98

2. Saad F, Lipton A, Cook R, Chen YM, Smith M, Coleman R (2007) Pathologic fractures correlate with reduced survival in patients with malignant bone disease. Cancer 110:1860-1867

3. Muramatsu K, Ihara K, Iwanagaa R, Taguchi T (2010) Treatment of metastatic bone lesions in the upper extremity: indications for surgery. Orthopedics 33:807

4. Ofluoglu O, Erol B, Ozgen Z, Yildiz M (2009) Minimally invasive treatment of pathological fractures of the humeral shaft. Int Orthop 33:707-712

5. Hansen BH, Keller J, Laitinen M et al (2004) The Scandinavian Sarcoma Group Skeletal Metastasis Register. Survival after surgery for bone metastases in the pelvis and extremities. Acta Orthop Scand Suppl 75:11-15

6. Cazzato RL, Palussière J, Buy X et al (2015) Percutaneous long bone cementoplasty for palliation of malignant lesions of the limbs: a systematic review. Cardiovasc Intervent Radiol 38:1563-1572

7. Lee C (2005) Properties of bone cement: the mechanical properties of PMMA bone cement. In: Breusch S, Malchau H (eds) The well-cemented total hip arthroplasty. Springer, Berlin, Heidelberg, pp 60-66

8. Cazzato RL, Buy X, Eker O, Fabre T, Palussiere J (2014) Percutaneous long bone cementoplasty of the limbs: experience with fifty-one non-surgical patients. Eur Radiol 24:3059-3068

9. Premat K, Clarençon F, Bonaccorsi R, Degos V, Cormier É, Chiras J (2017) Reinforced cementoplasty using dedicated spindles in the management of unstable malignant lesions of the cervicotrochanteric region. Eur Radiol 27:3973-3982

10. Kelekis A, Filippiadis D, Anselmetti $G$ et al (2016) Percutaneous augmented peripheral osteoplasty in long bones of oncologic patients for pain reduction and prevention of impeding pathologic fracture: the rebar concept. Cardiovasc Intervent Radiol 39:90-96

11. Liu XW, Jin P, Liu K et al (2016) Comparison of percutaneous long bone cementoplasty with or without embedding a cement-filled catheter for painful long bone metastases with impending fracture. Eur Radiol 27:120-127

12. Tian $\mathrm{QH}, \mathrm{He}$ CJ, Wu CG et al (2016) Comparison of percutaneous cementoplasty with and without interventional internal fixation for impending malignant pathological fracture of the proximal femur. Cardiovasc Intervent Radiol 39:81-89

13. He C, Tian Q, Wu CG, Gu Y, Wang T, Li M (2014) Feasibility of percutaneous cementoplasty combined with interventional internal fixation for impending pathologic fracture of the proximal femur. J Vasc Interv Radiol 25:1112-1117

14. Cazzato RL, Garnon J, Tsoumakidou G et al (2017) Percutaneous imageguided screws mediated osteosnthesis of impending and pathological/ insufficiency fractures of the femoral neck in non-surgical cancer patients. Eur J Radiol 90:1-5

15. Lin PP, Kang HG, Kim Yl, Kim JH, Kim HS (2015) Minimally invasive surgery for femoral neck fractures using bone cement infusible hollow-perforated screw in high-risk patients with advanced cancer. Surg Oncol 24:226-231

16. Cornelis FH, Tselikas L Carteret T et al (2017) Percutaneous internal fixation with Y-STRUT ${ }^{\circledR}$ device to prevent both osteoporotic and pathological hip fractures: a prospective pilot study. J Orthop Surg Res 12:27

17. Kim JH, Kang HG, Kim JR, Lin PP, Kim HS (2011) Minimally invasive surgery of humeral metastasis using flexible nails and cement in high-risk patients with advanced cancer. Surg Oncol 20:32-37

18. Kim YI, Kang HG, Kim TS, Kim JH, Kim HS (2014) Palliative percutaneous stabilization of lower extremity for bone metastasis using flexible nails and bone cement. Surg Oncol 23:192-198

19. Müller ME, Nazarian S, Koch P, Schatzker J (1990) The comprehensive classification of fractures of long bones. Springer-Verlag, Berlin

20. Beckmann J, Springorum R, Vettorazzi E et al (2011) Fracture prevention by femoroplasty--cement augmentation of the proximal femur. J Orthop Res 29:1753-1758

21. Springorum HR, Gebauer M, Mehrl A et al (2014) Fracture prevention by prophylactic femoroplasty of the proximal femur--metallic compared with cemented augmentation. J Orthop Trauma 28:403-409 
22. Varga P, Inzana JA, Schwiedrzik J et al (2017) New approaches for cementbased prophylactic augmentation of the osteoporotic proximal femur provide enhanced reinforcement as predicted by non-linear finite element simulations. Clin Biomech (Bristol, Avon) 44:7-13

23. Palumbo BT, Nalley C, Gaskins RB 3rd et al (2014) Biomechanical analysis of impending femoral neck fractures: the role of percutaneous cement augmentation for osteolytic lesions. Clin Biomech (Bristol, Avon) 29:289-295

24. Szpalski M, Gunzburg R, Aebi M et al (2015) A new approach to prevent contralateral hip fracture: evaluation of the effectiveness of a fracture preventing implant. Clin Biomech (Bristol, Avon) 30:713-719

25. Nakata K, Kawai N, Sato M et al (2010) Percutaneous osteoplasty with a bone marrow nail for fractures of long bones: experimental study. J Vasc Interv Radiol 21:1436-1441

26. Nakata K, Kawai N, Sato M et al (2011) Bone marrow nails created by percutaneous osteoplasty for long bone fracture: comparisons among acrylic cement alone, acrylic-cement-filled bare metallic stent, and acryliccement-filled covered metallic stent. Cardiovasc Intervent Radiol 34:609-614

27. Yurdoglu C, Orsel S, Canibeyaz M, Kutuk O, Ucisik AH (1997) Augmentation of cement by $\mathrm{K}$ wires in filling the defect of bone. Acta Orthop Traumatol Turc 31:250-254

28. Green JK, Werner FW, Dhawan R, Evans PJ, Kelley S, Webster DA (2005) A biomechanical study on flexible intramedullary nails used to treat pediatric femoral fractures. J Orthop Res 23:1315-1320

29. Ramseier LE, Janicki JA, Weir S, Narayanan UG (2010) Femoral fractures in adolescents: a comparison of four methods of fixation. J Bone Joint Surg Am 92:1122-1129

30. Milin L, Sirveaux F, Eloy F, Mainard D, Molé D, Coudane H (2014) Comparison of modified Hackethal bundle nailing versus anterograde nailing for fixation of surgical neck fractures of the humerus: retrospective study of 105 cases. Orthop Traumatol Surg Res 100:265-270

31. Willeumier JJ, van der Linden YM, van de Sande MAJ, Dijkstra PDS (2016) Treatment of pathological fractures of the long bones. EFORT Open Rev 1:136-145

32. Hunt KJ, Gollogly S, Randall RL (2006) Surgical fixation of pathologic fractures. An evaluation of evolving treatment methods. Bull Hosp Jt Dis 63:77-82

33. Hong J, Cabe GD, Tedrow JR, Hipp JA, Snyder BD (2004) Failure of trabecular bone with simulated lytic defects can be predicted noninvasively by structural analysis. J Orthop Res 22:479-486

34. Sutter EG, Mears SC, Belkoff SM (2010) A biomechanical evaluation of femoroplasty under simulated fall conditions. J Orthop Trauma 24:95-99

\section{Submit your manuscript to a SpringerOpen ${ }^{\circ}$ journal and benefit from:}

- Convenient online submission

- Rigorous peer review

- Open access: articles freely available online

- High visibility within the field

- Retaining the copyright to your article

Submit your next manuscript at $>$ springeropen.com 